\title{
INDEX TO VOLUME 15
}

Aberbach, Ian M., and Li, Jinjia Asymptotic vanishing conditions which force regularity in local rings of prime characteristic ............... 815

Ahlgren, Scott, and Boylan, Matthew Odd coefficients of weakly holomorphic modular forms ....................................... 409

Akhmedov, Anar, Etnyre, John B., Mark, Thomas E., and Smith, Ivan A note on Stein fillings of contact manifolds ................ 1127

Alkan, Emre, Xiong, Maosheng, and Zaharescu, Alexandru A bias phenomenon on the behavior of Dedekind sums ................... 1039

Apostolov, Vestislav, and Dloussky, Georges Bihermitian metrics on Hopf surfaces ....................................... 827

Aramayona, Javier, Parlier, Hugo, and Shackleton, Kenneth J. Totally geodesic subgraphs of the pants complex ....................... 309

Arone, Greg, Lambrechts, Pascal, Turchin, Victor, and Volić, Ismar Coformality and rational homotopy groups of spaces of long knots ...... 1

Baldwin, John A. Comultiplicativity of the Ozsváth-Szabó contact invariant 273

Baranovsky, Vladimir A universal enveloping for $L_{\infty}$-algebras .......... 1073

Barreto, Antônio Sá A Support Theorem For The Radiation Fields On Asymptotically Euclidean Manifolds ............................ 973

Bateman, Michael, and Katz, Nets Hawk Kakeya sets in Cantor

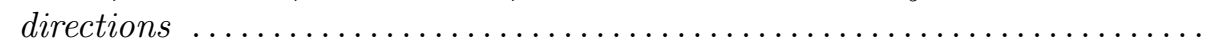

Benguria, Rafael D., Frank, Rupert L., and Loss, Michael The sharp constant in the Hardy-Sobolev-Maz'ya inequality in the three dimensional upper half-space ...................................... 613

Biquard, Olivier Continuation unique à partir de l'infini conforme pour les métriques d'Einstein ....................................... 1091

Bivià-Ausina, Carles Joint reductions of monomial ideals and multiplicity of complex analytic maps .................................... 389

Blache, Régis, Férard, Éric, and Zhu, Hui June Hodge-Stickelberger polygons for L-functions of exponential sums of $P\left(x^{s}\right) \ldots \ldots \ldots \ldots \ldots 1053$

Blair, Matthew D., Smith, Hart F., and Sogge, Christopher D. On multilinear spectral cluster estimates for manifolds with boundary ....... 419

Borell, Stefan, Kutzschebauch, Frank, and Wold, Erlend Fornæss Proper holomorphic disks in the complement of varieties in $\mathbb{C}^{2} \ldots \ldots . .821$

Borisov, Lev, and Libgober, Anatoly Higher elliptic genera ......... 511

Boylan, Matthew see Ahlgren, Scott, and Boylan, Matthew ...... 409

Brenner, Holger, and Kaid, Almar On deep Frobenius descent and flat bundles .............................................. 1101

Buczyński, Jarosław Hyperplane sections of Legendrian subvarieties . . . . 623

Burns, David On refined Stark conjectures in the non-abelian case ...... 841

Carles, Rémi, and Ozawa, Tohru On the wave operators for the critical nonlinear Schrödinger equation ............................ 185 
Carro, María J. On the hypothesis $K^{*}$ of Hardy, Littlewood and Hooley and its relation with discrete fractional operators ................ 1117

Caviglia, Giulio, and Maclagan, Diane Some cases of the Eisenbud-GreenHarris conjecture ................................. 427

Chen, Jiun-Cheng, and Tseng, Hsian-Hua A note on derived McKay

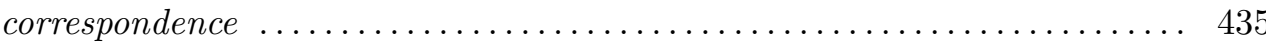

Cleyton, Richard, and Ivanov, Stefan Conformal equivalence between

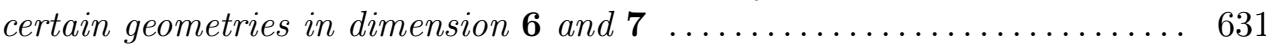

Clop, Albert, and Tolsa, Xavier Analytic capacity and quasiconformal mappings with $W^{1,2}$ Beltrami coefficient .................. 779

Coates, Tom Givental's Lagrangian cone and $S^{1}$-Equivariant Gromov-Witten

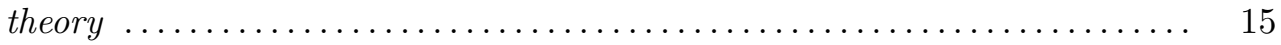

Dao, Hailong Some observations on local and projective hypersurfaces . . . . 207

David, Chantal, and Weston, Tom Local torsion on elliptic curves and the deformation theory of Galois representations .................. 599

Diaz, Francisco Diaz y, and Friedman, Eduardo Finite products of regu-

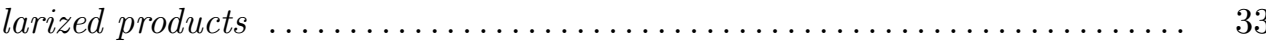

Dloussky, Georges see Apostolov, Vestislav, and Dloussky, Georges 827

Duyckaerts, Thomas, Holmer, Justin, and Roudenko, Svetlana Scattering for the non-radial 3D cubic nonlinear Schrödinger equation . . . . . 1233

Escauriaza, L., Kenig, C.E., Ponce, G., and Vega, L. Convexity properties of solutions to the free Schrödinger equation with Gaussian decay ... 957

Etingof, Pavel, and Gelaki, Shlomo Quasisymmetric and unipotent tensor

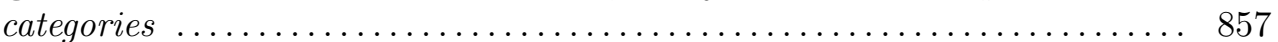

Etnyre, John B. see Akhmedov, Anar, Etnyre, John B., Mark, Thomas E., and Smith, Ivan ..................... 1127

Férard, Éric see Blache, Régis, Férard, Éric, and Zhu, Hui June . . 1053

Fang, Fuquan Positive Quaternionic Kähler manifolds and symmetry rank:

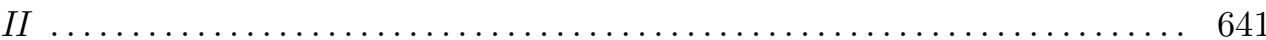

Fasel, J., and Srinivas, V. A vanishing theorem for oriented intersection

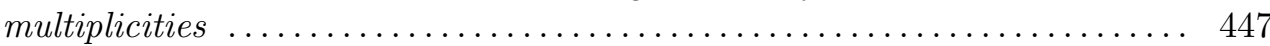

Frank, Rupert L. see Benguria, Rafael D., Frank, Rupert L., and

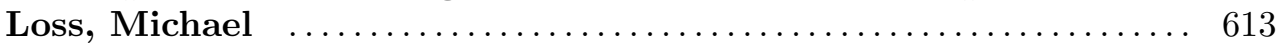

Frenkel, Péter E. Pfaffians, hafnians and products of real linear functionals 351

Friedlander, John B., Kurlberg, Pär, and Shparlinski, Igor E. Products

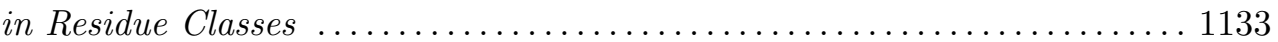

Friedman, Eduardo see Diaz, Francisco Diaz y, and Friedman, Ed-

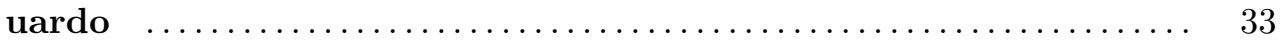

Garthwaite, Sharon Anne, and Penniston, David p-adic properties of Maass forms arising from theta series ...................... 459

Gautam, S. Zubin A critical-exponent Balian-Low theorem .......... 471

Gekhtman, M. I., and Vaninsky, K. L. The family of analytic Poisson brackets for the Camassa-Holm hierarchy .................. 867

Gekhtman, Michael, Shapiro, Michael, and Vainshtein, Alek On the properties of the exchange graph of a cluster algebra .............. 321

Gelaki, Shlomo see Etingof, Pavel, and Gelaki, Shlomo _...... 857 
Ghioca, Dragos Mordell exceptional locus for subvarieties of the additive

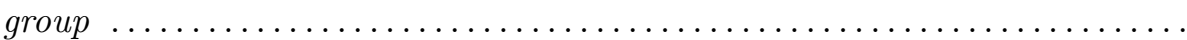

González-Avilés, Cristian D. Algebraic cycles on Severi-Brauer schemes of prime degree over a curve ............................. 51

González-Avilés, Cristian D. Chevalley's ambiguous class number formula for an arbitrary torus . ............................. 1149

Goto, Shiro, Nishida, Koji, and Ozeki, Kazuho The structure of Sally modules of rank one ................................. 881

Greenblatt, Michael Simply nondegenerate multilinear oscillatory integral

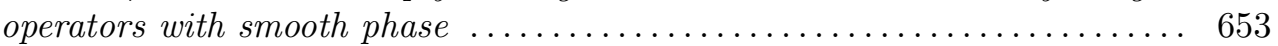

Harada, Megumi, and Landweber, Gregory D. The K-theory of abelian

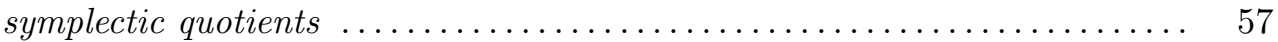

Harrington, Phillip S. The order of Plurisubharmonicity on pseudoconvex domains with Lipschitz boundaries ...................... 485

Holmer, Justin see Duyckaerts, Thomas, Holmer, Justin, and Roudenko,

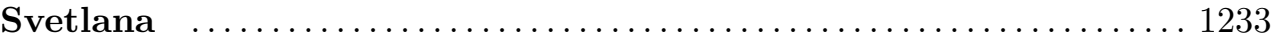

Howe, E. W., Maisner, D., Nart, E., and Ritzenthaler, C. Principally polarizable isogeny classes of abelian surfaces over finite fields . . . . . . . . 121

Hur, Vera Mikyoung Symmetry of solitary water waves with vorticity . . . 491

Ivanov, Stefan see Cleyton, Richard, and Ivanov, Stefan . . . . .. 631

Jenkinson, Oliver $A$ partial order on $\times 2$-invariant measures $\ldots . \ldots \ldots \ldots 89$

Jow, Shin-Yao, and Miller, Ezra Multiplier ideals of sums via cellular resolutions .............................................. 359

Kaid, Almar see Brenner, Holger, and Kaid, Almar ........... 1101

Katz, Nets Hawk see Bateman, Michael, and Katz, Nets Hawk . . . 73

Kaufmann, Ralph M. A proof of a cyclic version of Deligne's conjecture via

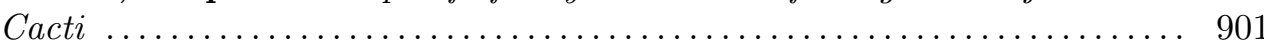

Kenig, C.E. see Escauriaza, L., Kenig, C.E., Ponce, G., and Vega,

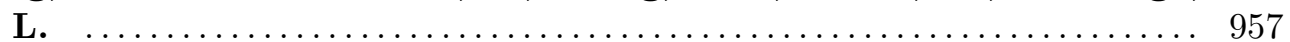

Kim, Byoung Du (B.D.) The algebraic functional equation of an elliptic

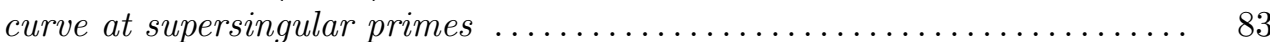

Koch, Herbert, Smith, Hart F., and Tataru, Daniel Subcritical $L^{p}$ bounds on spectral clusters for Lipschitz metrics . ................ 993

Koskela, Pekka, and Saksman, Eero Pointwise characterizations of Hardy-

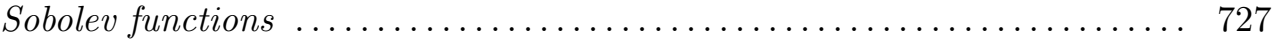

Kucharz, W. Curves on algebraic models of smooth manifolds . . . . . ... 289

Kurlberg, Pär see Friedlander, John B., Kurlberg, Pär, and Shpar-

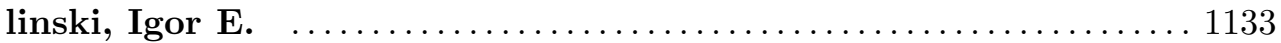

Kutzschebauch, Frank see Borell, Stefan, Kutzschebauch, Frank, and Wold, Erlend Fornæss $\ldots \ldots \ldots \ldots \ldots \ldots \ldots \ldots \ldots \ldots \ldots \ldots \ldots \ldots \ldots \ldots \ldots$

Lai, BaiShun, and Li, Yi On the semistability of the minimal positive steady state for a nonhomogeneous semilinear Cauchy problem ............. 923

Lam, Kwan-hang Spectrum of the Laplacian on manifolds with Spin(9) ho-

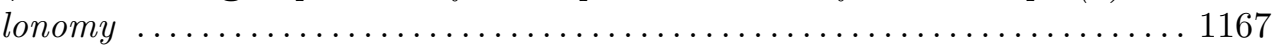

Lambrechts, Pascal see Arone, Greg, Lambrechts, Pascal, Turchin, Victor, and Volić, Ismar 
Landweber, Gregory D. see Harada, Megumi, and Landweber, Gregory $\mathbf{D}$.

Lassalle, Michel A positivity conjecture for Jack polynomials ........... 661

Lee, Yng-Ing, and Wang, Mu-Tao A note on the stability and uniqueness for solutions to the minimal surface system .......................

Leung, Naichung Conan, and Li, Changzheng Hard Lefschetz actions in Riemannian geometry with special holonomy ................... 683

Li, Changzheng see Leung, Naichung Conan, and Li, Changzheng . 683

Li, Jinjia see Aberbach, Ian M., and Li, Jinjia $\ldots \ldots \ldots \ldots \ldots \ldots . \ldots 15$

$\mathbf{L i}$, Yi see Lai, BaiShun, and Li, Yi . ..................... 923

Libgober, Anatoly see Borisov, Lev, and Libgober, Anatoly ...... 511

Long, D. D., and Reid, A. W. Finding fibre faces in finite covers ..... 521

Loss, Michael see Benguria, Rafael D., Frank, Rupert L., and Loss, Michael ........................................ 613

Maclagan, Diane see Caviglia, Giulio, and Maclagan, Diane ..... 427

Maisner, D. see Howe, E. W., Maisner, D., Nart, E., and Ritzenthaler,

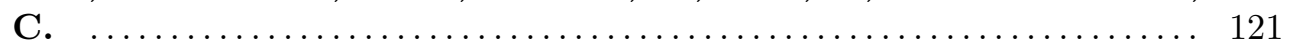

Mark, Thomas E. see Akhmedov, Anar, Etnyre, John B., Mark, Thomas E., and Smith, Ivan $\ldots \ldots \ldots \ldots \ldots \ldots \ldots \ldots \ldots \ldots \ldots \ldots \ldots \ldots \ldots$

Migliore, Juan, Nagel, Uwe, and Zanello, Fabrizio A characterization of Gorenstein Hilbert functions in codimension four with small initial degree 331

Miller, Ezra see Jow, Shin-Yao, and Miller, Ezra _............. 359

Momot, Aleksander Irregular ball-quotient surfaces with non-positive Kodaira dimension ....................................... 1187

Morishita, Masanori, and Terashima, Yuji Geometry of polysymbols . . 95

Moskowitz, Martin, and Sacksteder, Richard On complex exponential groups .............................................. 1197

Nagel, Uwe see Migliore, Juan, Nagel, Uwe, and Zanello, Fabrizio . 331

Nart, E. see Howe, E. W., Maisner, D., Nart, E., and Ritzenthaler,

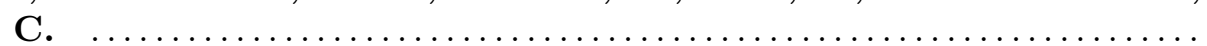

Nazarov, F., Treil, S., and Volberg, A. Two weight inequalities for individual Haar multipliers and other well localized operators ............. 583

Ni, Lei, and Wallach, Nolan On a classification of gradient shrinking soli-

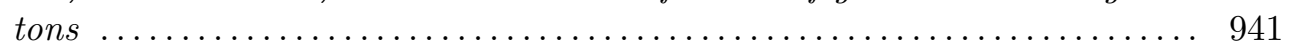

Nishida, Koji see Goto, Shiro, Nishida, Koji, and Ozeki, Kazuho . 881

Ozawa, Tohru see Carles, Rémi, and Ozawa, Tohru ............. 185

Ozeki, Kazuho see Goto, Shiro, Nishida, Koji, and Ozeki, Kazuho . 881

Panyushev, Dmitri I., and Yakimova, Oksana S. The argument shift method and maximal commutative subalgebras of Poisson algebras ...... 239

Papikian, Mihran Modular curves of $\mathcal{D}$-elliptic sheaves are asymptotically

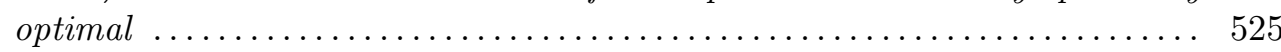

Pappas, Georgios Grothendieck-Riemann-Roch and the moduli of Enriques

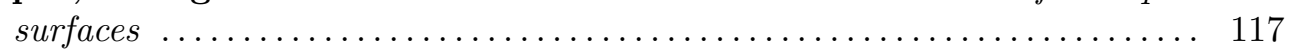

Parlier, Hugo see Aramayona, Javier, Parlier, Hugo, and Shackleton, Kenneth J. 
Pauly, Christian On the base locus of the linear system of generalized theta functions ............................................ 699

Penniston, David see Garthwaite, Sharon Anne, and Penniston, David ............................................ 459

Petrović, Sonja On the Universal Gröbner bases of varieties of minimal

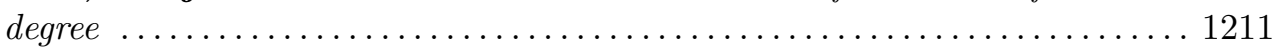

Polishchuk, A. Fourier-stable subrings in the Chow rings of abelian

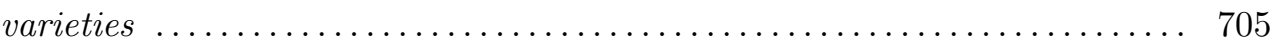

Ponce, G. see Escauriaza, L., Kenig, C.E., Ponce, G., and Vega, L. 957

Poonen, Bjorn Smooth hypersurface sections containing a given subscheme over a finite field ..................................... 265

Raich, Andrew S., and Straube, Emil J. Compactness of the complex Green operator ..................................... 761

Rasmussen, Christopher, and Tamagawa, Akio A finiteness conjecture on abelian varieties with constrained prime power torsion ............. 1223

Reid, A. W. see Long, D. D., and Reid, A. W. ............... 521

Ritter, Jürgen, and Weiss, Alfred Congruences between abelian pseudomea-

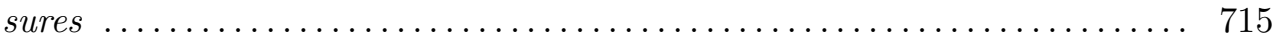

Ritzenthaler, C. see Howe, E. W., Maisner, D., Nart, E., and Ritzen-

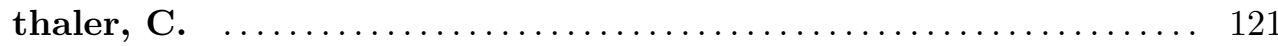

Rotger, Victor Which quaternion algebras act on a modular abelian

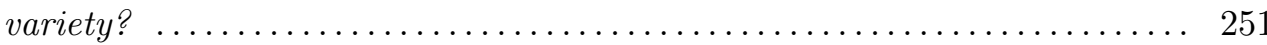

Roudenko, Svetlana see Duyckaerts, Thomas, Holmer, Justin, and

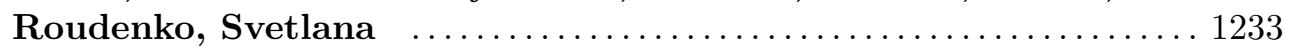

Sacksteder, Richard see Moskowitz, Martin, and Sacksteder, Rich-

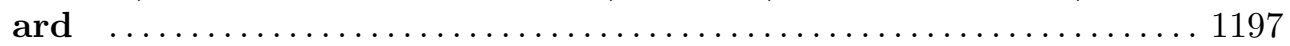

Saksman, Eero see Koskela, Pekka, and Saksman, Eero _......... 727

Schein, Michael M. On modular weights of Galois representations ....... 537

Schwede, Karl Generalized test ideals, sharp F-purity, and sharp test elements ............................................. 1251

Shackleton, Kenneth J. see Aramayona, Javier, Parlier, Hugo, and Shackleton, Kenneth J. ............................. 309

Shapiro, Ilya, and Tang, Xiang Gelfand-Fuchs cohomology of invariant formal vector fields ....................................... 129

Shapiro, Michael see Gekhtman, Michael, Shapiro, Michael, and Vain-

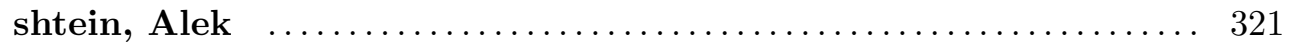

Shparlinski, Igor E. see Friedlander, John B., Kurlberg, Pär, and Shparlinski, Igor E. . ............................ 1133

Smith, Ivan see Akhmedov, Anar, Etnyre, John B., Mark, Thomas E., and Smith, Ivan .............................. 1127

Smith, Hart F. see Blair, Matthew D., Smith, Hart F., and Sogge, Christopher D. ................................. 419

Smith, Hart F. see Koch, Herbert, Smith, Hart F., and Tataru,

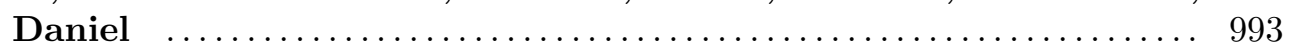

Sogge, Christopher D. see Blair, Matthew D., Smith, Hart F., and Sogge, Christopher D. ............................... 419 


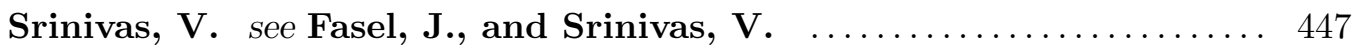

Stokman, Jasper V. Generalized Cherednik-Macdonald identities ....... 745

Straube, Emil J. see Raich, Andrew S., and Straube, Emil J. . ... 761

Sturmfels, Bernd, and Tevelev, Jenia Elimination theory for tropical varieties .............................................. 543

Sun, Zhi-Wei An additive theorem and restricted sumsets ............ 1263

Szymik, Markus Stable Diffeomorphism Groups of 4-Manifolds ......... 1003

Takagi, Shunsuke, and Takahashi, Ryo D-modules over rings with finite F-representation type ................................... 563

Takahashi, Ryo see Takagi, Shunsuke, and Takahashi, Ryo ....... 563

Tamagawa, Akio see Rasmussen, Christopher, and Tamagawa, Akio 1223

Tang, Xiang see Shapiro, Ilya, and Tang, Xiang _.............. 129

Tataru, Daniel see Koch, Herbert, Smith, Hart F., and Tataru, Daniel ........................................... 993

Tawn, Stephen A presentation for Hilden's subgroup of the braid group . . . 1277

Terashima, Yuji see Morishita, Masanori, and Terashima, Yuji .... 95

Tevelev, Jenia see Sturmfels, Bernd, and Tevelev, Jenia ......... 543

Thomas, Hugh, and Yong, Alexander An $S_{3}$-symmetric Littlewood-Richardson rule ........................................... 1027

Tolsa, Xavier see Clop, Albert, and Tolsa, Xavier _............. 779

Treil, S. see Nazarov, F., Treil, S., and Volberg, A. ............ 583

Tseng, Hsian-Hua see Chen, Jiun-Cheng, and Tseng, Hsian-Hua . 435

Turchin, Victor see Arone, Greg, Lambrechts, Pascal, Turchin, Victor,

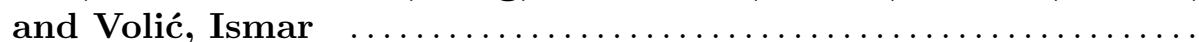

Ulrich, Bernd, and Validashti, Javid A criterion for integral dependence of modules ............................................... 149

Vainshtein, Alek see Gekhtman, Michael, Shapiro, Michael, and Vainshtein, Alek ................................... 321

Validashti, Javid see Ulrich, Bernd, and Validashti, Javid ....... 149

Vaninsky, K. L. see Gekhtman, M. I., and Vaninsky, K. L. . . . . . 867

Vega, L. see Escauriaza, L., Kenig, C.E., Ponce, G., and Vega, L. . . 957

Viale, Matteo A family of covering properties ..................... 221

Volberg, A. see Nazarov, F., Treil, S., and Volberg, A. .......... 583

Volić, Ismar see Arone, Greg, Lambrechts, Pascal, Turchin, Victor,

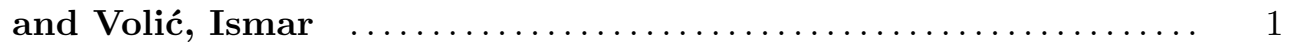

Vu, Van H. Sum-product estimates via directed expanders ............. 375

Wakefield, Max, and Yoshinaga, Masahiko The Jacobian ideal of a hyperplane arrangement .................................... 795

Wallach, Nolan see Ni, Lei, and Wallach, Nolan ................ 941

Wang, Mu-Tao see Lee, Yng-Ing, and Wang, Mu-Tao ........... 197

Weiss, Alfred see Ritter, Jürgen, and Weiss, Alfred ............ 715

Wenger, Stefan Characterizations of metric trees and Gromov hyperbolic spaces ............................................ 1017

Weston, Tom see David, Chantal, and Weston, Tom ............ 599

Wold, Erlend Fornæss see Borell, Stefan, Kutzschebauch, Frank, and Wold, Erlend Fornæss . ................................... 821 
Xiong, Maosheng see Alkan, Emre, Xiong, Maosheng, and Zaharescu, Alexandru ...................................... 1039

Yakimova, Oksana S. see Panyushev, Dmitri I., and Yakimova, Oksana S. ............................................. 239

Yong, Alexander see Thomas, Hugh, and Yong, Alexander ....... 1027 Yoshinaga, Masahiko see Wakefield, Max, and Yoshinaga, Masahiko ......................................... 795

Zaharescu, Alexandru see Alkan, Emre, Xiong, Maosheng, and Za-

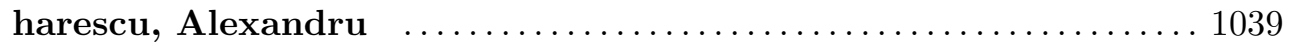

Zanello, Fabrizio see Migliore, Juan, Nagel, Uwe, and Zanello, Fab-

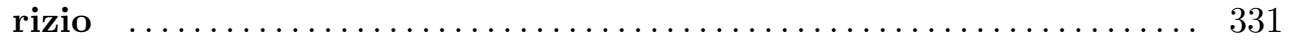

Zhang, R.B. see Zhang, Guanglian, and Zhang, R.B. .......... 297

Zhang, De-Qi Automorphism groups and anti-pluricanonical curves ...... 163

Zhang, Guanglian, and Zhang, R.B. Equivariant vector bundles on quantum homogeneous spaces .................................. 297

Zhang, Jing Algebraic Stein Varieties .......................... 801

Zhu, Hui June see Blache, Régis, Férard, Éric, and Zhu, Hui June 1053 